\title{
Accurate Extraction of Equivalent Circuit Parameters of Spiral Resonators for the Design of Metamaterials
}

\author{
Danilo Brizi, Student Member, IEEE, Nunzia Fontana, Filippo Costa, Member, IEEE, and Agostino \\ Monorchio, Fellow, IEEE
}

\begin{abstract}
Spiral resonators are one of the most common typology of resonant magnetic unit cell for the realization of metamaterials. The precise knowledge of their lumped electric properties (RLC parameters) is of crucial importance in the metamaterial design. Thus, an accurate and unambiguous procedure for estimating the value of the RLC lumped parameters of compact spiral resonators is introduced. The proposed procedure relies on a rigorous approach allowing a complete characterization of spiral resonators also in terms of $Q$ factor. The method is general and valid for other shapes of resonators. The estimations have been finally verified by performing measurements on fabricated spiral resonators through a magnetic probe.
\end{abstract}

Index Terms - Metamaterial, resonant frequency, scattering parameters, spiral resonators, unit cell.

\section{INTRODUCTION}

$\mathrm{M}^{\mathrm{s}}$ ETAMATERIALS are nowadays a consolidated branch of electromagnetic research and they are aimed at providing a novel class of artificial engineered materials able to show anomalous properties, not present in natural materials. Metamaterials can provide negative values of complex dielectric permittivity and magnetic permeability [1]-[4]. These properties are achieved over a narrow frequency bands and they are due to resonant behavior of miniaturized resonators [5], [6]. Because of the small footprint of the elementary resonators compared to the wavelength, various homogenization approaches [7]-[10] have been proposed to interpret these particles or array of particles as bulk material with negative permittivity or permeability values. Various resonators shapes have been proposed in the literature to extremely stress the miniaturization of the particle with respect to the operating

Manuscript received June 6, 2018; revised September 1, 2018; accepted October 31, 2018.

D. Brizi, F. Costa and A. Monorchio are with the Department of Information Engineering, University of Pisa, 56122 - Pisa, Italy (e-mail: danilo.brizi@ing.unipi.it,_filippo.costa@iet.unipi.it, agostino.monorchio@unipi.it).

N. Fontana is with Consorzio Nazionale Interuniversitario per le Telecomunicazioni (CNIT), 56122 - Pisa, Italy (e-mail: nunzia.fontana@cnit.it). wavelength [11]-[13]. Among this broad class of materials, Spiral Resonators (SRs) have demonstrated their ability to provide a high level of miniaturization and they have been extensively used as metamaterial unit cell or as decoupling distributed filter in array applications [14]-[16]. Moreover, SRs have been recently employed also in the fast-growing field of the energy harvesting, because of their property to act as an inductive tank circuit able to store energy coming from the environmental electromagnetic pollution [17]-[19].

A key aspect in the design of a spiral resonator is to derive an accurate equivalent circuit representation of the miniaturized inclusion thus avoiding long and timeconsuming electromagnetic simulations. A number of works in literature reports helpful analytical model of passive spiral resonators (SRs) [5], [20]-[22]. The SR is usually modelled as a RLC series resonator and the values of the $\mathrm{L}$ and $\mathrm{C}$ parameters are derived starting from classical electrostatic considerations [5]. Such models revealed themselves able to provide a correct estimation of the resonance frequency compared to simulations [5] and measurements [22]. The experimental verification of the resonance frequency is usually carried out by employing two monopoles closely located to the resonator [22]. However, a rigorous verification of the values of the estimated $\mathrm{L}$ and $\mathrm{C}$ parameters used to characterize the resonator is not available. In particular, a different behavior of the values of capacitance and inductance as a function of the number of spiral turns have been observed by using different models [5], [20], [23] even with a similar estimation of the resonance frequency. As it is well known, there exist infinite couples of $\mathrm{L}$ and $\mathrm{C}$ that provide a certain resonance frequency for an LC circuit; in this sense, a complete characterization is achieved only if the derived $\mathrm{L}$ and $\mathrm{C}$ values are verified against an accurate estimation obtained, for instance, from a full-wave simulation [24], [25]. The measurement of the resonators with one or two external antennas permits only the estimation of the resonance frequency since the coupling between the interrogating antenna and the passive resonator is unknown.

As a consequence, a reliable procedure, based on full-wave simulations or measured data, which guarantee the accurate extraction of the RLC parameters without any ambiguity is 
certainly missing in the literature. Not only the resonance frequency has to be predicted by the RLC parameters, but also the behavior of the resonator around the resonance frequency. In this sense, the extraction procedure is the new contribution of this paper to such important issue.

The $\mathrm{R}, \mathrm{L}$ and $\mathrm{C}$ parameters of the circuit model are obtained by including the RLC series circuit within an accurate circuit model of the entire simulation set-up. Once that the simulation setup is accurately characterized (including the mutual coupling between the interrogating antenna and the spiral resonators), the RLC parameters remain the sole unknows and they can be precisely and unambiguously derived by using a fitting procedure. In this way, the actual RLC values as a function of the number of turns can also be obtained and interesting considerations about the electromagnetic behavior of the spiral resonators can be performed, including a proper estimation of the Qfactor. Our approach is completely general, and it allows analyzing any shape of resonators, unlike the fully-analytical works developed in the literature, in most cases suitable only for particular spiral resonators.

The paper is organized as follows. Section II is focused on the description of the simulation setup used to estimate the lumped RLC parameters of the spiral resonators. Some results in terms of extraction of RLC parameters for different resonators are reported in Section III. In Section IV, we develop some physical considerations about the obtained characterization results. The attention is posed on the behavior of $\mathrm{L}$ and $\mathrm{C}$ values as a function of the number of turns. Finally, in Section $\mathrm{V}$, measured results aimed at verifying the correct estimation of the resonance frequency and of the equivalent RLC circuit of the spiral resonators with our simulation set-up are presented. Conclusions follow.

\section{Procedure For The Estimation Of Rlc Parameters OF THE SPIRAL RESONATOR}

The goal of the paper is to introduce a reliable extraction procedure for RLC parameters of a resonant magnetic inclusion as a spiral resonator. A lumped model with constant parameter values describes correctly the SR behavior especially in the proximity of its resonance point, which is the region of utmost importance in order to design metamaterials.

The estimation of the $\mathrm{R}, \mathrm{L}, \mathrm{C}$ parameters of the spiral resonator is performed by using the simulation set-up depicted in Fig. 1a. It consists of the probe loop and the spiral resonator (SR) under test. The probe loop is non-resonating and fed by coaxial cable. The SR is placed at the center of the probe loop. The simulation setup shown in Fig. 1a is schematized by the equivalent circuit proposed in Fig. 1c [26], [27]. The external probe loop is represented by the $R_{\text {loop }}$ and $L_{\text {loop }}$ due to its inductive nature. The SR is represented by the RLC series circuit on the right-hand part. The coupling coefficient $M_{\text {loopSR }}$ takes into account the mutual coupling between the two circuits. A block diagram of the proposed procedure is reported in Fig. 2. The characterization process of the loop resonator starts with a full-wave simulation (or measurement) of the $S_{11}$ of the probe loop standalone. The simulations, in our case, has been performed through CST Studio Suite (CST Computer Simulation Technology AG, Darmstadt, Germany). Once calculated the $S_{11}$ of the probe, the input impedance, $Z_{11}$, is straightforwardly computed. At this point the parameters $R_{\text {loop, }} L_{\text {loop }}$ as a function of frequency are computed. This step is required in order to completely characterize the simulation setup, leaving the SR's RLC parameters as the unique unknowns. The SR under test is therefore placed centered with respect to the probe loop and the mutual impedance $M_{\text {loopSR }}$ value must be determined.

The mutual impedance value $M_{\text {loopSR }}$ quantifies the amplitude of the inductive coupling between the two elements. This parameter can be estimated through a magneto-static approach [28], once the geometrical parameters of the set-up are known.

Indeed, under quasi-static hypothesis, it is possible to apply Biot-Savart formulation to estimate the mutual coupling between two generic coils. This assumption is substantiated by the small dimension of the set-up with respect to the wavelength and its geometrical properties. The typical dimension of the considered spiral resonators is around $1 \mathrm{~cm}$ whereas the wavelength at $300 \mathrm{MHz}$ (in the middle of the chosen frequency span) is equal to $1 \mathrm{~m}$ in the vacuum. Considering a quasi-static assumption valid until the resonator is smaller than one tenth of the applied wavelength, we can set an upper bound for our hypothesis at around $3 \mathrm{GHz}$.

Thus, the magnetic field produced by a generic current path at a generic point can be expressed as:

$$
\vec{B}(\vec{r})=\frac{\mu_{0}}{4 \pi} \int \frac{I \overrightarrow{d l} \times \vec{r}}{\left.\overrightarrow{\mid r^{\prime}}\right|^{3}}
$$

where $\mu_{0}(\mathrm{H} / \mathrm{m})$ is the magnetic permeability of the vacuum, $I$ (A) is the current amplitude flowing in the path, $\overrightarrow{d l}(\mathrm{~m})$ is an infinitesimal element of the current path and $\vec{r}(\mathrm{~m})$ is the distance between a generic point of the space and the infinitesimal element $\overrightarrow{d l}$.

The mutual coupling coefficient between a generic coil $j$ and a coil $i$ is defined as the magnetic flux ( $\left.\Phi_{i j}\right)$ through the coil $j$ induced by the current flowing in the coil $i$ :

$$
M_{i j}=\frac{\Phi_{i j}}{I_{i}}
$$

Afterwards, supposing a unit current in the coil $i\left(I_{i}\right)$, the mutual coefficient $M_{i j}$ is simply the flux of the magnetic field analytically calculated from (1) through the surface of the coil $j$.

In this way, given the geometrical properties of the two elements constituting the system, we can numerically set a unit current flowing in one of the two coils and evaluate the inductive mutual coupling on the remaining one. Obviously, it 


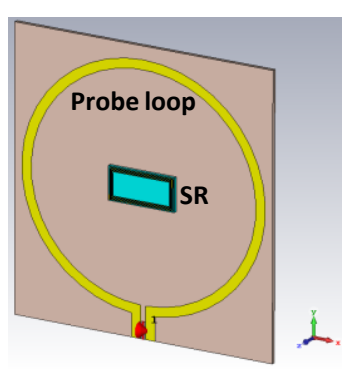

(a)

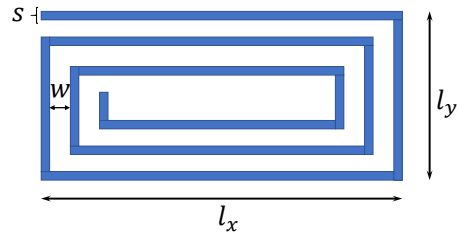

(b)

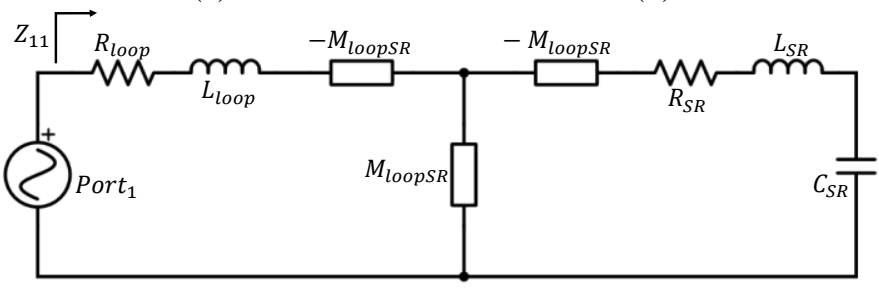

(c)

Fig. 1. (a) CAD model of the adopted simulation set-up (CST Studio Suite, Darmstadt). (b) Representation of a generic spiral resonator (drawing is not in scale). (c) Equivalent lumped circuit for inductively coupled spiral resonator and probe loop.

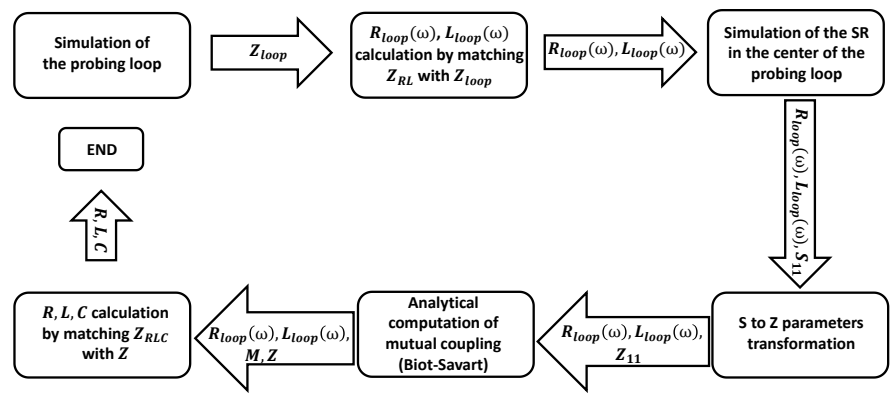

Fig. 2. Block diagram of the proposed retrieving method.

is also valid that $M_{i j}=M_{j i}$. Thus, a value of the mutual coefficient expressed in $\mathrm{nH}$ can be obtained.

At this point, the only unknown parameters in the model of Fig. 1c are the RLC parameters of the spiral resonator.

In order to find the most suitable values of the RLC circuit, we implemented in a computer code the lumped circuit of Fig. $1 \mathrm{c}$; therefore, it was possible to evaluate the $Z_{11}$ parameter according to the lumped elements values:

$$
\begin{aligned}
& Z_{11}(\omega)=\left[\left(R_{S R}+j \omega L_{S R}+1 / j \omega C_{S R}-j \omega M_{\text {loopSR }}\right)\right. \\
& \left.\| j \omega M_{\text {loopSR }}\right]+R_{\text {loop }}+j \omega L_{\text {loop }}-j \omega M_{\text {loopSR }}
\end{aligned}
$$

Since $R_{\text {loop }}, L_{\text {loop }}$ and $M_{\text {loopSR }}$ parameters are known, (3) is a function of the SR's lumped electric parameters only.

At this point, we compare the $Z_{11}$ obtained from full-wave simulations (i.e. $Z_{11_{\text {CST }}}$ in (4)) with the $Z_{11}$ expressed in (3) (i.e. $\left.Z_{11_{\text {Fititin }}}\right)$. Among all the infinite combinations of RLC parameters for the spiral resonator producing a resonance at the frequency estimated by the full-wave software, there will be one that better fits the simulated $Z_{11}$; in particular, a mean root square cost function has been chosen:

$$
\begin{aligned}
& \min \{\text { err }\}=\min \left\{\sqrt{\sum_{\omega}\left\{\left[\operatorname{Im}\left(Z_{11_{\text {CST }}}\right)-\operatorname{Im}\left(Z_{11_{\text {Fiting }}}\right)\right]^{2}+\right.}\right. \\
& \left.\left.+\left[\operatorname{Re}\left(Z_{11_{\text {CST }}}\right)-\operatorname{Re}\left(Z_{11_{\text {Fiting }}}\right)\right]^{2}\right\}\right\}
\end{aligned}
$$

As previously stated, the resonant frequency $f_{\text {res }}$ of the spiral resonator can be easily detected from the full wave simulation. As the resonant properties of the spiral resonator are only dependent on its $\mathrm{L}$ and $\mathrm{C}$ values, we choose the best fitting LC couple satisfying the following relation:

$$
f_{\text {res }}=\frac{1}{2 \pi \sqrt{L C}}
$$

On the other hand, the resistance term $\mathrm{R}$ influences the Qfactor of the spiral resonator and it is spanned in the fitting procedure independently from the $\mathrm{L}$ and $\mathrm{C}$ values.

In order to obtain physically meaningful initial seeds for the fitting procedure, we apply the method presented in [29]. By exploiting the model described in (3), we carried out a deembedding procedure on the simulated $Z_{11_{\text {CST }}}$ in order to get the RLC parameters of the spiral. From (3), the de-embedded parameters can be calculated as:

$$
\left(R_{S R}+j \omega L_{S R}+1 / j \omega C_{S R}\right)=\frac{-\left(j \omega M_{\text {loop } S R}\right)^{2}}{Z_{11}(\omega)-Z_{\text {loop }}}
$$

The real part of this function evaluated at the resonant frequency gives the initial seed for the resistance.

$$
R_{S R_{\text {sece }}}=\Re\left\{\frac{-\left(j \omega_{\text {res }} M_{\text {loop } S R}\right)^{2}}{Z_{11}\left(\omega_{\text {res }}\right)-Z_{\text {loop }}\left(\omega_{\text {res }}\right)}\right\}
$$

On the other hand, the half of the derivative of the imaginary component of (6) (i.e., LC series), always evaluated at the resonant frequency, gives the initial seed for $\mathrm{L}$.

$$
\begin{aligned}
& L_{S R_{\text {sead }}}=\left.\frac{1}{2} \frac{\partial}{\partial \omega}\left\{\mathfrak{\Im}\left\{R_{S R}+j \omega L_{S R}+1 / j \omega C_{S R}\right\}\right\}\right|_{\omega=\omega_{\text {res }}}= \\
& =\left.\frac{1}{2} \frac{\partial}{\partial \omega}\left\{\mathfrak{J}\left\{\frac{-\left(j \omega M_{\text {loop } S R}\right)^{2}}{Z_{11}(\omega)-Z_{\text {loop }}(\omega)}\right\}\right\}\right|_{\omega=\omega_{\text {res }}}
\end{aligned}
$$

The initial seed for C simply follows from (5).

In this way, we can obtain the combination of RLC parameters that better fits the full-wave simulation, thus providing an unambiguous characterization of the SR under test.

\section{NumericAl ReSUltS}

We performed a series of tests of the proposed fitting procedure in order to characterize the behavior of different spiral resonators (SRs) as a function of the number of turns. 


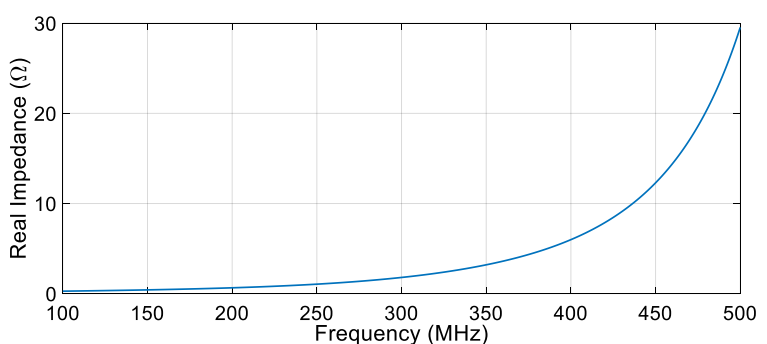

(a)

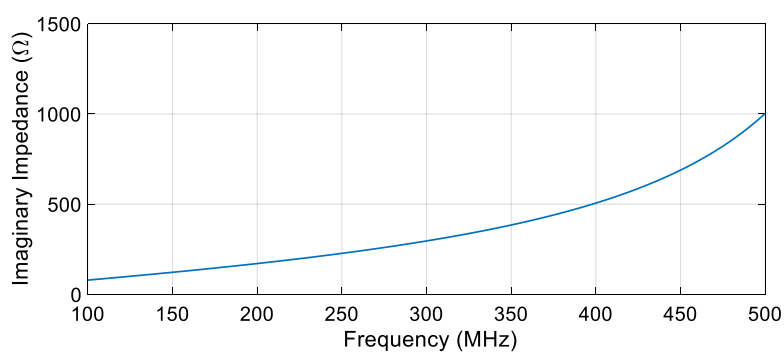

(b)

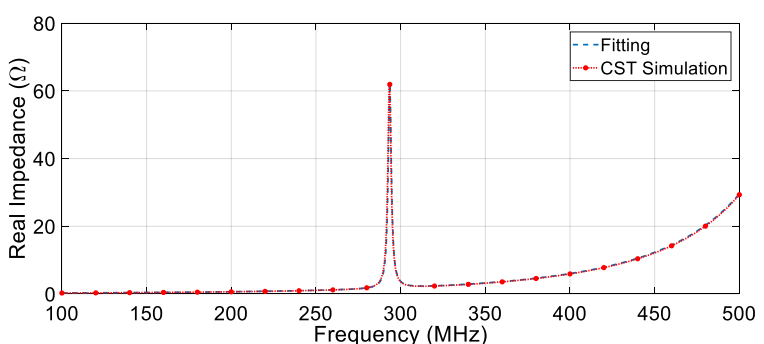

(c)

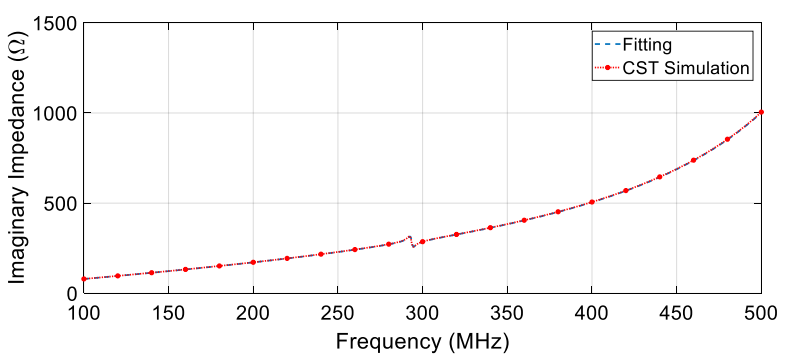

(d)

Fig. 3. Impedance of the loop (i.e. the larger magnetic probe) without the spiral resonator: (a) Real part, (b) Imaginary part. Impedance of the probing loop coupled with one of the spiral resonators $\left(N=6, l_{x}=13.7 \mathrm{~mm}, l_{y}=6.7 \mathrm{~mm}\right)$ with the fitting obtained by using the lumped equivalent circuit in Fig. $1 \mathrm{c}$ : (c) Real part, (d) Imaginary part. It can be noted that the resonant frequency of the spiral resonator is easily detectable from the full-wave simulation.

TABLE I

Analyzed Seiral Resonators With Our FitTing Procedure

\begin{tabular}{ccccc}
\hline \hline Shape & $N$ & $w=s(\mathrm{~mm})$ & $l_{x}(\mathrm{~mm})$ & $l_{y}(\mathrm{~mm})$ \\
\hline Rectangular & {$[3,4,5,6,7,8,9$,} & 0.127 & 13.7 & 6.7 \\
Square & $10,11,12,13]$ & & & \\
& {$[3,5,7,9,11,13]$} & 0.127 & 6.7 & 6.7 \\
\hline \hline
\end{tabular}

In particular, we selected the meaningful cases summarized in Table I. They consisted in two different shapes for spiral resonators (square and rectangular, respectively) and we computed the $\mathrm{R}, \mathrm{L}$ and $\mathrm{C}$ with respect to the number of turns $N$.

The probe loop was non-resonant, and it was not loaded with any reactive loads. The loop was made of copper and it was etched on a $0.8 \mathrm{~mm}$ thick FR4 substrate $\left(\varepsilon_{r}=4.3, \tan \delta=0.025\right)$. As a first step, we characterized the probe loop standalone in order to obtain its proper selfimpedance (Fig. 1a). For the case of the larger rectangular spiral resonator, it consisted in a loop of $5 \mathrm{~cm}$ diameter, with a strip width of $2 \mathrm{~mm}$. The frequency span was set between 50 and $500 \mathrm{MHz}$. On the other hand, we selected a smaller probe loop for the square resonator $(2 \mathrm{~cm}$ diameter), in order to enhance the mutual coupling between the probe and the spiral (whose area is smaller with respect to the rectangular one), spanning the spectrum between $250 \mathrm{MHz}$ and $1 \mathrm{GHz}$. The behavior of this probe loop was similar to the case of the larger probe but shifted in frequency. In order to clarify the effect of the spiral resonator placed in the middle of the probe loop, we reported in Fig. 3 both the impedance of the unloaded probe (real and imaginary part) and its impedance loaded with a spiral resonator with the following parameters: $N=6, l_{x}=13.7$ $\mathrm{mm}, l_{y}=6.7 \mathrm{~mm}$. A similar behavior is observed for other resonators with different dimensions. As expected, the real component of the impedance of the probe loop increases with the frequency, due to the more pronounced skin effect (Fig. 3a). In the same way, we can see that the imaginary component is totally inductive (Fig. 3b), as predictable, because we added no reactive load to the probe. Thus, we concluded that the probe loop is effectively representable as a $R_{\text {loop }}, L_{\text {loop }}$ equivalent circuit.

In general, a spiral resonator can be defined as a N-turns planar spiral, presenting different shapes, strip width $(s)$ and gap between strips $(w)$ (Fig. 1b). For simplicity, we selected these two parameters as equal in our tests.

In Fig. 3 (c and d) we reported the real and imaginary components of the impedance of the probe loop inductively coupled with the rectangular spiral resonator ( $N=6$ turns, lateral dimensions of $l_{x}=13.7 \mathrm{~mm}$ and $l_{y}=6.7 \mathrm{~mm}$ ). As it is apparent, both the real and the imaginary components of the $Z_{11}$ were well fitted by the proposed procedure, with an almost perfect overlap between the simulated and the fitted curves. This confirmed the validity of the employed circuit model for the probe-spiral resonator system.

The spiral resonators were made of copper and etched on an $0.8 \mathrm{~mm}$ thick Arlon substrate $\left(\varepsilon_{r}=3.58, \tan \delta=0.0035\right)$. This substrate is small compared to the probe substrate, and it is placed, centered, on the top of the probe loop.

Once that the geometrical properties of the system are known (probe loop and the particular spiral resonator under test), as described in the previous Section, it was possible to apply Biot-Savart approximation in order to evaluate the mutual coupling. It must be noted, as shown in Fig. 4, that the estimated mutual coupling coefficients correctly increase with the number of turns of the spirals. Indeed, each turn added to 


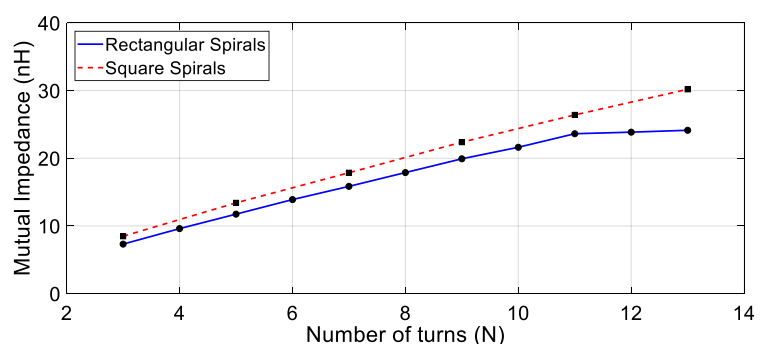

Fig. 4. Mutual impedance between the considered rectangular spiral resonators (aspect ratio: 2.04) and the larger probe loop (solid line); Mutual impedance between the considered square spiral resonators and the smaller probe loop (dashed line).

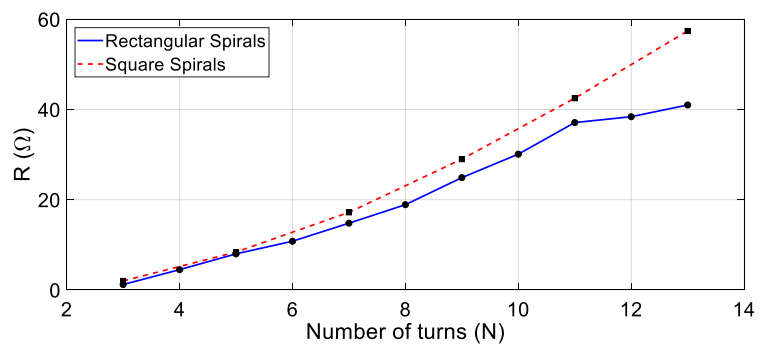

(a)

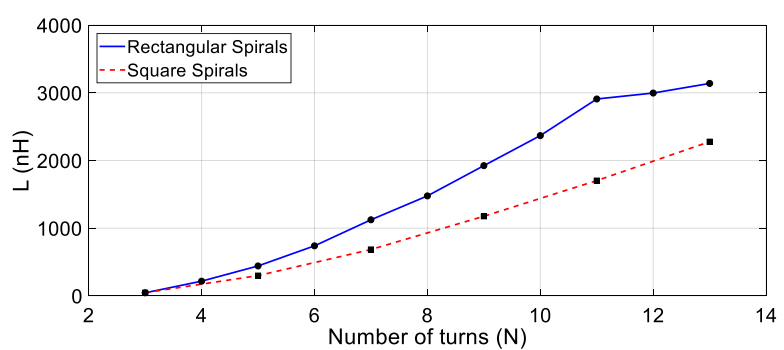

(b)

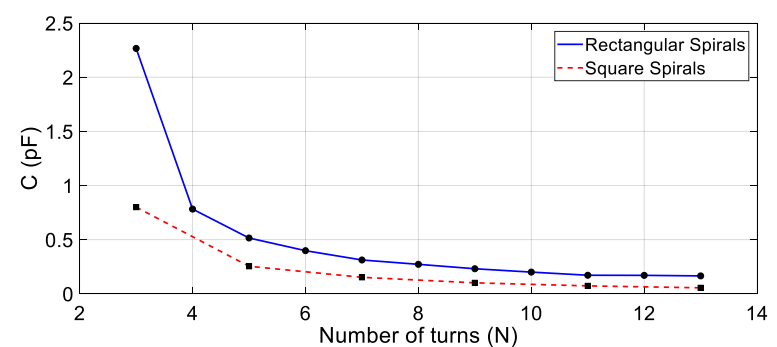

(c)

Fig. 5. Impedance of the spiral resonators (rectangular and square) obtained from the fitting when the number of turns increases: (a) Resistance, (b) Inductance, (c) Capacitance.

the spiral resonator increases the area available for the linkage of the magnetic field produced by the fed probe loop.

At this point, the only undetermined unknowns remained the RLC parameters of the specific SR under test. By using a specifically designed Matlab algorithm, the RLC parameters were derived, according to (3) and (4), for each spiral configuration reported in Table I. We exploited the initial RLC seeds derived according (7) and (8).

As already stated, it is interesting at this point to evaluate the behavior of the $\mathrm{R}, \mathrm{L}$ and $\mathrm{C}$ of the spiral resonator as a function of the number of turns when the external dimensions of the spiral are fixed. Fig. 5 shows the behavior of the RLC

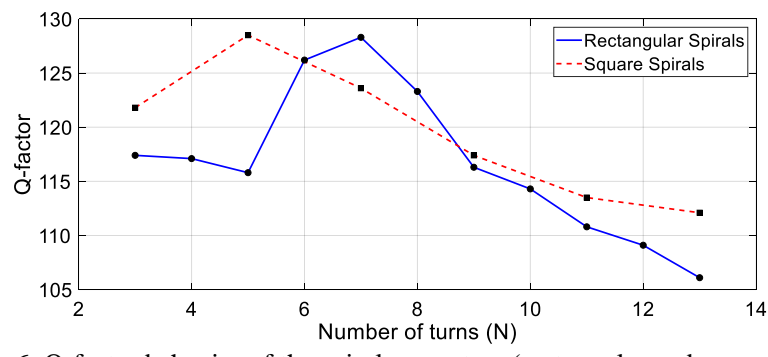

Fig. 6. Q-factor behavior of the spiral resonators (rectangular and square) with the increasing number of turns.

TABLE II

Analyzed Rectangular Siral Resonators With Our Fitting Procedure

\begin{tabular}{ccccccc}
\hline \hline$N$ & $R(\Omega)$ & $L(\mathrm{nH})$ & $C(\mathrm{pF})$ & $Q$-factor & $\begin{array}{c}R \text { seed } \\
(\Omega)\end{array}$ & $\begin{array}{c}\text { L seed } \\
(\mathrm{nH})\end{array}$ \\
\hline 3 & 1.2 & 45 & 2.266 & 117.4 & 1.2 & 45 \\
4 & 4.5 & 217 & 0.782 & 117.1 & 4.5 & 217 \\
5 & 8.0 & 442 & 0.515 & 115.8 & 7.9 & 443 \\
6 & 10.8 & 739 & 0.398 & 126.2 & 10.6 & 744 \\
7 & 14.8 & 1124 & 0.312 & 128.3 & 14.8 & 1129 \\
8 & 18.9 & 1477 & 0.272 & 123.3 & 18.8 & 1491 \\
9 & 24.9 & 1924 & 0.231 & 116.3 & 24.2 & 1928 \\
10 & 30.1 & 2368 & 0.200 & 114.3 & 29.9 & 2392 \\
11 & 37.1 & 2909 & 0.171 & 110.8 & 36.9 & 2929 \\
12 & 38.4 & 2997 & 0.170 & 109.1 & 38.3 & 2994 \\
13 & 41.0 & 3139 & 0.165 & 106.1 & 40.8 & 3132 \\
\hline \hline
\end{tabular}

TABLE III

ANAlyzed SQuared Siral Resonators With Our Fitting Procedure

\begin{tabular}{ccccccc}
\hline \hline$N$ & $R(\Omega)$ & $L(\mathrm{nH})$ & $C(\mathrm{pF})$ & $Q$-factor & $\begin{array}{c}R \text { seed } \\
(\Omega)\end{array}$ & $\begin{array}{c}\text { L seed } \\
(\mathrm{nH})\end{array}$ \\
\hline 3 & 2.0 & 47 & 0.800 & 121.8 & 1.9 & 48 \\
5 & 8.4 & 299 & 0.254 & 128.5 & 8.3 & 310 \\
7 & 17.2 & 685 & 0.152 & 123.6 & 16.9 & 689 \\
9 & 29.0 & 1176 & 0.101 & 117.4 & 28.6 & 1187 \\
11 & 42.5 & 1703 & 0.073 & 113.5 & 42.3 & 1705 \\
13 & 57.4 & 2278 & 0.055 & 112.1 & 56.9 & 2267 \\
\hline \hline
\end{tabular}

parameters for the previously fitted spirals (rectangular and square) when the number of turns spanned as described in Table I. Once computed the actual RLC parameters, it is also possible to derive the Q-factor of the spiral resonator, which is important for correctly describing the selectivity of the resonance.

Q-factor can be typically described as [30]:

$$
Q=\frac{\omega_{r e s} L}{R}=\frac{1}{R} \sqrt{\frac{L}{C}}
$$

where $\omega_{\text {res }}$ is the resonant frequency whereas $R, L, C$ are the extracted electrical lumped parameters of the considered spiral resonator. As shown in Fig. 6, the Q-factor for the rectangular SR increases as a function of the number of turns up to a particular value, when it starts to diminish. The same analysis was performed also for the square spiral resonator. The results of the estimated lumped RLC parameters and the Q-factor are finally summarized in Table II and Table III, along with the 
comparison with the initial seeds estimated from the deembedded simulated impedance of each spiral resonators.

\section{PhysicAl CONSIDERATIONS ON EXTRACTED PARAMETERS}

The results obtained through the fitting procedure are well suited for a physical interpretation.

First of all, it can be seen from Fig. 5 that the resistance term increases almost linearly with the increase of the turns. This effect is expected because each turn added to the spiral resonator raises the total copper length and, thus, the losses in the spiral. In the same way, the inductance presents an approximately quadratic behavior with the number of turns which is meaningful for spiral-shaped object [31]-[34].

More importantly, the behavior of the inductance (as well as the resistance) of the spiral resonators show a saturation with the increase of the number of turns; this can be easily explained by the increasing filling factor of the spiral area (i.e. each added turn becomes smaller than the previous) that is more evident for the rectangular spiral rather than for the square shape. This result is opposite to that presented by [22], [5]; the fully analytical model therein developed presents an inductance that decreases with the increase of the number of turns. However, our fitting procedure demonstrated an opposite behavior, which is in accordance with the classical physical background of the phenomenon.

Moreover, the behavior of the retrieved capacitance is clearly decreasing with the number of turns. Such behavior is compatible with a distributed capacitance obtained by the summation of capacitors in series. Again, our result is opposite to what developed in the literature through only analytical formulation [22], [5].

Finally, Fig. 6 describes the Q-factor of the considered spiral resonator with the increase of the number of turns. As it can be observed, there is a particular value of the number of turns, which brings to the maximum of the Q-factor. This is due to the simultaneous behavior of the resistance and the inductance of the spiral resonator (see (9)). When the number of turns is raising beyond a certain value, the total losses introduced in the spiral become predominant over the inductance increase. Such information is very important from a design point of view [35], [36], especially when the selectivity of the SR's resonance is a fundamental feature to obtain. It is finally worth to underline that our proposed procedure takes into account the presence of dielectrics by incorporating the effect on the losses and the distributed capacitance of its presence inside the extracted parameters.

\section{EXPERIMENTAL RESULTS}

We performed also experimental tests on fabricated spiral resonators: three different rectangular spirals and one square spiral resonator. A summary of the four investigated structures is shown in Fig. 7. The geometrical properties are reported in Table IV. The chosen dielectric substrate is a $0.8 \mathrm{~mm}$ thick Arlon substrate $\left(\varepsilon_{r}=3.58, \tan \delta=0.0035\right)$.

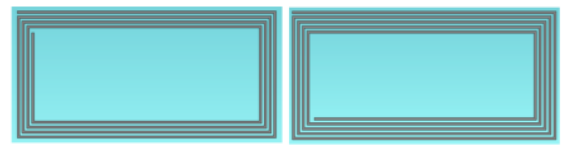

(a)

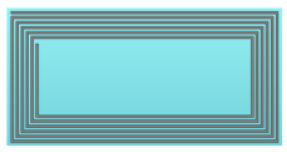

(c) (b)

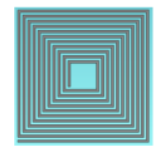

(d)
Fig. 7. Spiral resonators numerical 3D CAD model: (a) Rectangular N=4; (b) Rectangular N=5; (c) Rectangular N=6; (d) Square N=11.

TABLE IV

Properties Of The Spiral Resonators In Fig. 7; Simulated And Measured Resonance Frequencies Are ShOWn For CoMPARISON

\begin{tabular}{ccccccc}
\hline \hline $\mathrm{N}$ & $\begin{array}{c}w=s \\
(\mathrm{~mm})\end{array}$ & $l_{x}(\mathrm{~mm})$ & $l_{y}(\mathrm{~mm})$ & $\begin{array}{c}f_{\text {sim }} \\
(\mathrm{MHz})\end{array}$ & $\begin{array}{c}f_{\text {meas }} \\
(\mathrm{MHz})\end{array}$ & $\begin{array}{c}\text { Relative } \\
\text { error } \\
(\%)\end{array}$ \\
\hline 4 & 0.127 & 13.7 & 6.7 & 386.8 & 378 & $2.3 \%$ \\
5 & 0.127 & 13.7 & 6.7 & 334 & 327 & $2.1 \%$ \\
6 & 0.127 & 13.7 & 6.7 & 293.6 & 289 & $1.6 \%$ \\
11 & 0.127 & 6.7 & 6.7 & 453 & 442 & $2.4 \%$ \\
\hline \hline
\end{tabular}

In order to test the spiral resonators, we fabricated also a probe loop characterized by the same dimensions as in Section III. The testing setup is shown in Fig. 8. We exploited the mutual coupling between the small nonresonating probe loop and the passive spiral unit cell to be measured.

The spiral resonator was accommodated exactly in the center of the probe loop. The loop was connected to the Vector Network Analyzer (Keysight E5071C-ENA) through a RF cable with a 50- $\Omega$ SMA connector. The resonant frequencies of the four investigated spiral resonators have been obtained by identifying the local minimum in the $S_{11}$ of the probe. Table IV reports the measured resonant frequencies of all the four investigated spiral resonators. We observed a very good agreement between the full-wave numerical result of the resonant frequency $\left(f_{\text {sim }}\right)$ and the measured frequency $\left(f_{\text {meas }}\right)$ with the proposed method. The relative error between the measurements and numerical simulations was always less than $3 \%$ for the considered spiral resonators samples, demonstrating the effectiveness of our approach. Analogously as in previous sections, also the measured $S_{11}$ could be used to quantify the RLC parameters of the spiral resonators in addition to the resonant frequency. We replicated the procedure followed for the full-wave simulations for the three rectangular spirals. The lumped parameters estimated through the fitting procedure (Table V) show the same trend obtained with the simulations when the number of turns increases (i.e. $\mathrm{R}$ and $\mathrm{L}$ increasing and $\mathrm{C}$ decreasing). In particular, Fig. 9 shows one of the reconstructed $S_{11}$ parameter starting from the experimentally extracted lumped RLC parameters $\left(N=6, l_{x}=13.7 \mathrm{~mm}, l_{y}=6.7\right.$ $\mathrm{mm})$. In addition, the figure reports also the de-embedded spiral impedance, fitted with our procedure. 


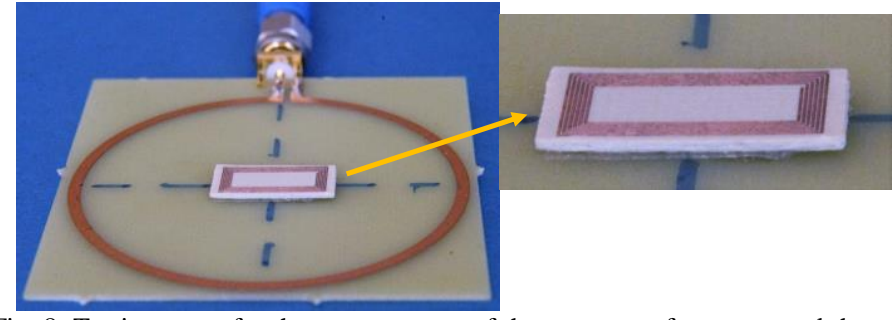

Fig. 8. Testing setup for the measurement of the resonance frequency and the impedance of the spiral resonator.

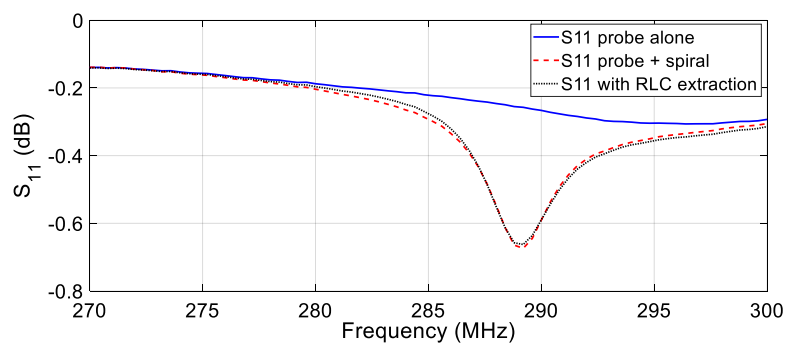

(a)

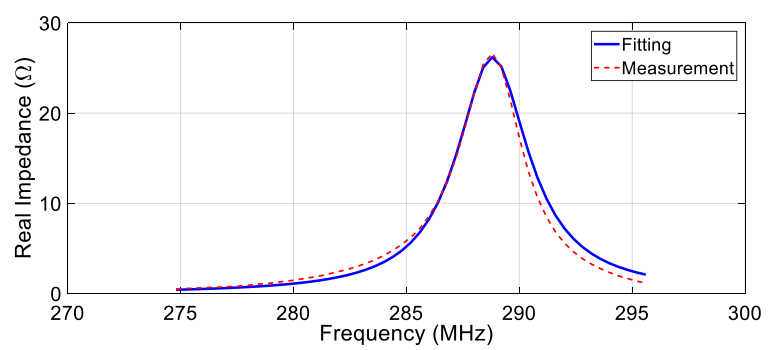

(b)

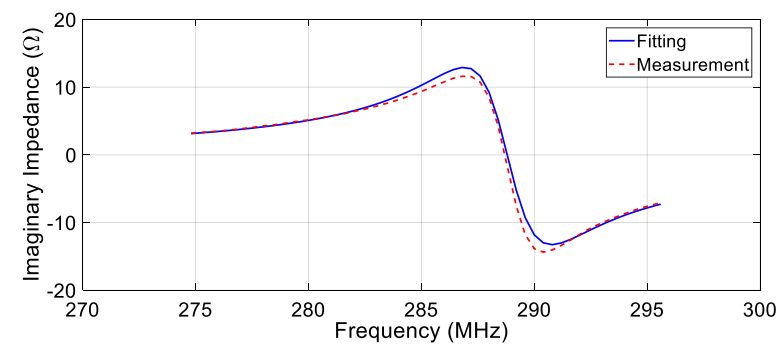

(c)

Fig. 9. (a) Comparison between measured $S_{11}$ of the larger probing loop with and without the rectangular spiral resonator $\left(N=6, l_{x}=13.7 \mathrm{~mm}, l_{y}=6.7\right.$ $\mathrm{mm})$ inside and $S_{11}$ reconstructed from the RLC parameters estimations (fitting); (b) and (c): Real and imaginary de-embedded measured spiral impedance and relative fitting results.

TABLE V

Obtained Lumped Parameters Of Rectangular SPIRAl Resonators: MEASUREMENTS (SIMULATIONS)

\begin{tabular}{ccccc}
\hline \hline$N$ & $R(\Omega)$ & $L(\mathrm{nH})$ & $C(\mathrm{pF})$ & $Q$-factor \\
\hline 4 & $7.3(4.5)$ & $142(217)$ & $1.251(0.782)$ & $46.2(117.1)$ \\
5 & $15.9(8.0)$ & $404(442)$ & $0.587(0.515)$ & $52.2(115.8)$ \\
6 & $21.6(10.8)$ & $910(739)$ & $0.334(0.398)$ & $76.5(126.2)$ \\
\hline \hline
\end{tabular}

Finally, it must be pointed out that we observed some variations of the RLC experimental values with respect to the full wave simulations; this can be addressed to the difficulties to realize a stable measurement environment (presence of losses and distortions). One direct effect of the fabrication of the prototype was the increase of the losses, due to soldering artifacts and connections. Moreover, the etching process can produce a pitting phenomenon, especially for extremely small width copper track as in this case, thus increasing the overall resistance of the path.

\section{CONCLUSION}

A novel accurate procedure for the extraction of the RLC parameters representing an isolated metamaterial spiral resonator has been introduced. We employed an experimental set-up consisting of a probe loop mutually coupled to the spiral resonator under test, which has been schematized through a suitable equivalent circuit topology. We firstly characterized the electromagnetic behavior of the stand-alone probe loop; then, following a magneto-static approach, we evaluated the mutual coupling coefficient existing between the probe and the spiral resonator under test. Finally, after a numerical simulation of the complete system, we extracted the $Z_{11}$ of the probe loop inductively coupled to the spiral resonator and we analytically matched the simulated impedance to the one of the lumped equivalent circuit, to retrieve RLC parameters of the spiral resonator. In this way, we obtained an accurate and unambiguous description of the electromagnetic properties of the spiral resonator. We also studied the variation of the RLC parameters and the Q-factor as a function of the shape (square or rectangular) and for different number of turns. We discussed the obtained results in terms of their physical meaning and we showed that a different behavior with respect to some popular models available in the literature has been observed. Finally, we performed measurements of some spiral resonators' shapes, by using a fabricated probe loop. The measured resonant frequencies of SRs are in good agreement with numerical simulation (relative error less than 3\%). We also accomplished the RLC parameters extraction from the measurements, obtaining a good correspondence with respect to the full-wave simulations, showing the consistency of our approach.

It must be worth noting that the developed procedure is completely general and can be applied to any typology of resonator, allowing a deeper understand and a more effective design of metamaterials.

\section{REFERENCES}

[1] J. B. Pendry, A. J. Holden, D. J. Robbins, and W. J. Stewart, "Magnetism from conductors and enhanced nonlinear phenomena," IEEE Trans. Microw. Theory Techn., vol. 47, no. 11, pp. 2075-2084, 1999.

[2] A. Sihvola, "Metamaterials in electromagnetics," Metamaterials, vol. 1, no. 1, pp. 2-11, Mar. 2007.

[3] S. A. Tretyakov, "Complex-media electromagnetics and metamaterials," J. Opt., vol. 19, no. 8, p. 084006, 2017.

[4] D. R. Smith, J. B. Pendry, and M. C. K. Wiltshire, "Metamaterials and negative refractive index," Science, vol. 305 , no. 5685, pp. 788792, Aug. 2004.

[5] F. Bilotti, A. Toscano, L. Vegni, K. Aydin, K. B. Alici, and E. Ozbay, "Equivalent-circuit models for the design of metamaterials based on artificial magnetic inclusions," IEEE Trans. Microw. Theory Techn., vol. 55, no. 12, pp. 2865-2873, Dec. 2007. 
[6] W. Withayachumnankul, C. Fumeaux, and D. Abbott, "Compact electric-LC resonators for metamaterials," Opt. Express, vol. 18, no. 25, pp. 25912-25921, Dec. 2010.

[7] C. R. Simovski, "Bloch material parameters of magneto-dielectric metamaterials and the concept of Bloch lattices," Metamaterials, vol. 1, no. 2, pp. 62-80, Dec. 2007.

[8] A. Alù, "First-principles homogenization theory for periodic metamaterials," Phys. Rev. B, vol. 84, no. 7, p. 075153, Aug. 2011.

[9] D. R. Smith and J. B. Pendry, "Homogenization of metamaterials by field averaging (invited paper)," JOSA B, vol. 23, no. 3, pp. 391-403, Mar. 2006.

[10] M. G. Silveirinha, "Metamaterial homogenization approach with application to the characterization of microstructured composites with negative parameters," Phys. Rev. B, vol. 75, no. 11, p. 115104, Mar. 2007.

[11] R. Marqués, F. Martin, and M. Sorolla, Metamaterials with Negative Parameters: Theory, Design, and Microwave Applications. John Wiley \& Sons, 2011.

[12] K. Aydin, I. Bulu, K. Guven, M. Kafesaki, C. M. Soukoulis, and E. Ozbay, "Investigation of magnetic resonances for different split-ring resonator parameters and designs," New J. Phys., vol. 7, no. 1, p. 168, 2005.

[13] K. B. Alici, F. Bilotti, L. Vegni, and E. Ozbay, "Optimization and tunability of deep subwavelength resonators for metamaterial applications: complete enhanced transmission through a subwavelength aperture," Opt. Express, vol. 17, no. 8, pp. 59335943, Apr. 2009

[14] I. R. O. Connell, K. M. Gilbert, M. A. Abou-Khousa, and R. S. Menon, "MRI RF array decoupling method with magnetic wall distributed filters," IEEE Trans. Med. Imag., vol. 34, no. 4, pp. 825835, Apr. 2015.

[15] I. R. O. Connell, K. M. Gilbert, M. A. Abou-Khousa, and R. S. Menon, "Design of a parallel transmit head coil at $7 \mathrm{~T}$ with magnetic wall distributed filters," IEEE Trans. Med. Imag., vol. 34, no. 4, pp. 836-845, Apr. 2015.

[16] N. Fontana, F. Costa, G. Tiberi, L. Nigro, and A. Monorchio, "Distributed trap FSS filter for dual tuned RF MRI coil decoupling at 7.0 T," in Electromagnetics in Advanced Appl. (ICEAA), 2017 Int. Conf. on, 2017, pp. 1229-1231.

[17] U. S. Pranav, S. Sudheesh, P. Stanly, S. Sankar, R. Devika, and A. Pradeep, "Metamaterial based energy harvester," Procedia Comput. Sci., vol. 93, pp. 74-80, 2016.

[18] T. S. Almoneef and O. M. Ramahi, "Metamaterial electromagnetic energy harvester with near unity efficiency," Appl. Phys. Lett., vol. 106, no. 15, p. 153902, Apr. 2015.

[19] C. Fowler and J. Zhou, "A metamaterial-inspired approach to RF energy harvesting," ArXiv Prepr. ArXiv170507718, 2017.

[20] J. D. Baena, R. Marqués, F. Medina, and J. Martel, "Artificial magnetic metamaterial design by using spiral resonators," Phys. Rev. $B$, vol. 69, no. 1, p. 014402, Jan. 2004.

[21] F. Falcone et al., "Stop-band and band-pass characteristics in coplanar waveguides coupled to spiral resonators," Microw. Opt. Technol. Lett., vol. 42, no. 5, pp. 386-388, Jul. 2004.

[22] F. Bilotti, A. Toscano, and L. Vegni, "Design of spiral and multiple split-ring resonators for the realization of miniaturized metamaterial samples," IEEE Trans. Antennas Propag., vol. 55, no. 8, pp. 22582267, Aug. 2007.

[23] H. Mosallaei and K. Sarabandi, "Magneto-dielectrics in electromagnetics: concept and applications," IEEE Trans. Antennas Propag., vol. 52, no. 6, pp. 1558-1567, Jun. 2004.

[24] F. Costa, A. Monorchio, and G. Manara, "Efficient analysis of frequency-selective surfaces by a simple equivalent-circuit model," IEEE Antennas Propag. Mag., vol. 54, no. 4, pp. 35-48, Aug. 2012.

[25] F. Costa, A. Monorchio, and G. Manara, "An overview of equivalent circuit modeling techniques of frequency selective surfaces and metasurfaces.," Appl. Comput. Electromagn. Soc. J., vol. 29, no. 12, 2014.

[26] B. Wu, X. Zhang, P. Qu, and G. X. Shen, "Design of an inductively decoupled microstrip array at 9.4T," J. Magn. Reson., vol. 182, no. 1, pp. 126-132, Sep. 2006
[27] J. Mispelter, M. Lupu, and A. Briguet, NMR Probeheads for Biophysical and Biomedical Experiments: Theoretical Principles \& Practical Guidelines. Imperial College Press, 2006.

[28] Y. Cheng and Y. Shu, "A new analytical calculation of the mutual inductance of the coaxial spiral rectangular coils," IEEE Trans. Magn., vol. 50, no. 4, pp. 1-6, 2014.

[29] G. Dambrine, A. Cappy, F. Heliodore, and E. Playez, "A new method for determining the FET small-signal equivalent circuit," IEEE Trans. Microw. Theory Techn., vol. 36, no. 7, pp. 1151-1159, Jul. 1988.

[30] R. E. Collin, Field Theory of Guided Waves 2nd edn. Piscataway, NJ: IEEE, 1990.

[31] D. Schieber, "On the inductance of printed spiral coils," Electr. Eng. Arch. Elektrotechnik, vol. 68, no. 3, pp. 155-159, 1985.

[32] H. A. Wheeler, "Simple inductance formulas for radio coils," Proc. Inst. Radio Eng., vol. 16, no. 10, pp. 1398-1400, 1928.

[33] S. S. Mohan, M. del Mar Hershenson, S. P. Boyd, and T. H. Lee, "Simple accurate expressions for planar spiral inductances," IEEE J. Solid-State Circuits, vol. 34, no. 10, pp. 1419-1424, 1999.

[34] F. W. Grover, Inductance calculations: working formulas and tables. Courier Corporation, 2004.

[35] G. Giovannetti, G. Tiberi, M. Tosetti, A. Monorchio, and N. Fontana, "Radiofrequency planar surface coil for magnetic resonance: When the use of a circular wire gives a noticeable advantage with respect to a flat strip conductor?," Measurement, vol. 129, pp. 518-522, Dec. 2018.

[36] G. Giovannetti, N. Fontana, A. Monorchio, M. Tosetti, and G. Tiberi, "Estimation of losses in strip and circular wire conductors of radiofrequency planar surface coil by using the finite element method," Concepts Magn. Reson. Part B Magn. Reson. Eng., vol. 47B, no. 3, p. e21358, Jul. 2017. 